\title{
Preface: Special issue dedicated to Distance Geometry
}

\author{
Farid Alizadeh ${ }^{1} \cdot$ Douglas Gonçalves $^{2} \cdot$ Nathan Krislock $^{3} \cdot$ Leo Liberti $^{4}$
}

Received: 16 July 2018 / Accepted: 24 July 2018 / Published online: 28 July 2018

(c) Springer Science+Business Media, LLC, part of Springer Nature 2018

\begin{abstract}
This preface introduces the special issue of the Journal of Global Optimization dedicated to the workshop "Distance Geometry Theory and Applications" (DGTA16) which took place at the DIMACS Center, Rutgers University, Piscataway NJ, USA.
\end{abstract}

Keywords Distance geometry $\cdot$ Inverse problem · Applied mathematics

Distance Geometry (DG) is based on distances rather than points and angles. The fundamental problem of DG is the DisTANCE GEOMETRY PROBLEM (DGP), which is an inverse problem to "given a set of points in $\mathbb{R}^{K}$, compute some of the pairwise distances". More precisely, given an integer $K>0$ and a simple undirected graph $G=(V, E)$ with a non-negative weight function $d: E \rightarrow \mathbb{R}_{+}$defined on the edges, it asks whether there exists a realization $x: V \rightarrow \mathbb{R}^{K}$ such that

$$
\forall\{i, j\} \in E \quad\left\|x_{i}-x_{j}\right\|=d_{i j} .
$$

DIMACS generously supported the Distance Geometry Theory and Applications workshop to which this special issue is dedicated. NSF support (through DIMACS) is also gratefully acknowledged.

$\bowtie$ Leo Liberti

liberti@lix.polytechnique.fr

Farid Alizadeh

farid.alizadeh@rutgers.edu

Douglas Gonçalves

douglas@mtm.ufsc.br

Nathan Krislock

nkrislock@niu.edu

1 Department of Management Science and Information Systems, Rutgers Center for Operations Research (RUTCOR), Rutgers University, 100 Rockafeller Road, Livingstone Campus, Piscataway, NJ 08854, USA

2 Departamento de Matemática, Universidade Federal de Santa Catarina, Florianópolis, Santa Catarina 88.040-900, Brazil

3 Department of Mathematical Sciences, Northern Illinois University, DeKalb, IL, USA

4 CNRS LIX École Polytechnique, 91128 Palaiseau, France 
In most applications, the norm $\|\cdot\|$ in Eq. (1) above is the Euclidean $\ell_{2}$ norm [34]. With this restriction, another informal description of the DGP is whether it is possible to draw a given weighted graph in $\mathbb{R}^{K}$ so that each edge $\{i, j\} \in E$ is drawn as a straight segment of length exactly $d_{i j}$. The DGP traditionally arises in several applications, e.g. clock synchronization protocols [41], localization of wireless sensors [3,4,8,22], protein conformation from Nuclear Magnetic Resonance (NMR) data $[6,12,19,26,30]$, control of autonomous underwater vehicles [5], rigidity of frameworks [2,18,43] and more [14].

DG occupies a well-established place in the history of mathematics [31]: examples are Heron's theorem [21], generalized by Cayley [10], Euler's conjecture [16] on the rigidity of polyhedra, followed by Cauchy's theorem for convex polyhedra [9] and Connelly's counterexample for nonconvex ones [11], Menger's first organized treatment of the subject [38], and Gödel's result for a simplex drawn on the sphere [17]. In most of the past history of DG the DGP only appears implicitly, and restricted to complete graphs. In other words, people looked at the correspondence between distances and points only when all possible distances between the points were known. While there are traces (e.g. in rigidity and statics [20,36,37]) of the importance of the DGP, it is not until much later, and presumably because of the increasing capabilities of computers, that the DGP was first formalized [44].

Ever since, the communities behind the science and engineering applications of DGP drove the study of this problem to a more prominent position within applied mathematics. Relatively recently, there was a realization that different application communities were replicating work already done in neighboring communities, and an attempt was made to re-unite the different communities working in DG. These attempts included the publication of surveys [27,28,34,35], the organization of workshops (the first Distance Geometry and Applications workshop in Manaus, Brazil in 2013 〈dga2013.icomp.ufam.edu.br/) [1]; the second DG workshop "Many Faces of Distances" in Campinas, Brazil in 2014 [24] /www.ime. unicamp.br/workshop_distances〉; the ICMS workshop on Geometric Rigidity in Edinburgh in 2016; the third Distance Geometry Theory and Applications workshop at DIMACS in 2016 〈dimacs.rutgers.edu/archive/Workshops/Distance), which this issue is dedicated to; the Distance Geometry Day in Rennes, France in 2016 〈forum.cs-dc.org/topic/517〉; the fourth Distance Geometry workshop at the Physikzentrum Bad Honnef, Germany, in 2017 (www. lix.polytechnique.fr/ liberti/dg17>; more workshops are currently being planned), and the publication of authored $[12,25,32]$ and edited $[39,43]$ books.

Two special issues of journals were dedicated to the DGTA16, one-this one-in the Journal of Global Optimization (JOGO) and another one in Discrete Applied Mathematics. Five articles were submitted to this issue and three of them were accepted for publication. The authors of three other articles on topics close to DG but submitted to normal JOGO issues were contacted, and a proposal was made to them to contribute their articles to this special issue: the proposal was accepted. This issue therefore consists of the following six articles:

1. Low-Rank Matrix Completion using Nuclear Norm Minimization and Facial Reduction, by S. Huang and H. Wolkowicz, which applies the facial reduction framework [23] to the minimization of the nuclear norm, a technique used to find low rank solutions of matrix completion problems;

2. The Minimum Distance Superset Problem: Formulations and Algorithms, by L. Fontoura, R. Martinelli, M. Poggi and T. Vidal, which proposes new formulations and techniques for a problem which is also known as partial digest problem [42], turnpike problem [13] and unassigned DGP (uDGP) [7,15]; 
3. A new algorithm for the small-field astrometric point-pattern matching problem, by C. Santiago, C. Lavor, S. Monteiro, A. Kroner-Martins, which proposes a new and rather exciting application of DG to make sense of astronomical data;

4. Minimal curvature-constrained networks, by D. Kirszenblat et al., which extends the DG input to include curvature information so that the edges will be drawn as curved, rather than straight, segments: the motivating application is underground mining;

5. Mixed integer quadratically-constrained programming model to solve the irregular strip packing problem with continuous rotations, by L. Cherri, A. Cherri and E. Soler, which describes formulations and methods for solving a two-dimensional cutting and packing problem with nonconvex polygonal pieces;

6. Tuning interval Branch-and-Prune for protein structure determination, by B. Worley et al., which presents the latest generation of Branch-and-Prune algorithms for the DGP $[29,33,40]$, with results in the determination of protein structure from NMR data.

The first three articles were actually accepted for this issue, while the last three were carried over from the queue of accepted papers in JOGO after asking the authors for permission.

We hope you enjoy this issue, and that you will develop an interest in DG.

The guest editors,

Farid Alizadeh, Piscataway

Douglas Gonçalves, Santa Catarina

Nathan Krislock, DeKalb

Leo Liberti, Palaiseau

\section{References}

1. Andrioni, A., Lavor, C., Liberti, L., Mucherino, A., Maculan, N., Rodriguez, R. (eds.): Proceedings of the workshop on distance geometry and applications. Universidade Federal do Amazonas, Manaus (2013)

2. Asimow, L., Roth, B.: The rigidity of graphs. Trans. Am. Math. Soc. 245, 279-289 (1978)

3. Aspnes, J., Eren, T., Goldenberg, D., Morse, S., Whiteley, W., Yang, R., Anderson, B., Belhumeur, P.: A theory of network localization. IEEE Trans. Mob. Comput. 5(12), 1663-1678 (2006)

4. Bachrach, J., Taylor, C.: Localization in sensor networks. In: Stojmenović, I. (ed.) Handbook of Sensor Networks, pp. 3627-3643. Wiley, New York (2005)

5. Bahr, A., Leonard, J., Fallon, M.: Cooperative localization for autonomous underwater vehicles. Int. J. Robot. Res. 28(6), 714-728 (2009)

6. Berger, B., Kleinberg, J., Leighton, T.: Reconstructing a three-dimensional model with arbitrary errors. J. ACM 46(2), 212-235 (1999)

7. Billinge, S., Duxbury, P., Gonçalves, D., Lavor, C., Mucherino, A.: Assigned and unassigned distance geometry: applications to biological molecules and nanostructures. 4OR-Q J Oper Res 14, 337-376 (2016)

8. Biswas, P., Lian, T., Wang, T., Ye, Y.: Semidefinite programming based algorithms for sensor network localization. ACM Trans. Sens. Netw. 2, 188-220 (2006)

9. Cauchy, A.L.: Sur les polygones et les polyèdres. J. de l'École Polytech. 16(9), 87-99 (1813)

10. Cayley, A.: A theorem in the geometry of position. Camb. Math. J. II, 267-271 (1841)

11. Connelly, R.: A counterexample to the rigidity conjecture for polyhedra. Publ. Mathématiques de l'IHES 47, 333-338 (1978)

12. Crippen, G., Havel, T.: Distance Geometry and Molecular Conformation. Wiley, New York (1988)

13. Dakić, T.: On the turnpike problem. Ph.D. thesis, Simon Fraser University (2000)

14. Dokmanić, I., Parhizkar, R., Ranieri, J., Vetterli, M.: Euclidean distance matrices: essential theory, algorithms and applications. IEEE Signal Process. Mag. 1053-5888, 12-30 (2015)

15. Duxbury, P., Granlund, L., Juhas, P., Billinge, S.: The unassigned distance geometry problem. Discret. Appl. Math. 204, 117-132 (2016)

16. Euler, L.: Continuatio fragmentorum ex adversariis mathematicis depromptorum: II Geometria, 97 . In: Fuss, P., Fuss, N. (eds.) Opera postuma mathematica et physica anno 1844 detecta, vol. I, pp. 494-496. Eggers \& C, Petropolis (1862) 
17. Gödel, K.: On the isometric embeddability of quadruples of points of $r_{3}$ in the surface of a sphere. In: Feferman, S., Dawson, J., Kleene, S., Moore, G., Solovay, R., van Heijenoort, J. (eds.) Kurt Gödel: Collected Works, vol. I, pp. 276-279. Oxford University Press, Oxford (1986)

18. Graver, J., Servatius, B., Servatius, H.: Combinatorial Rigidity. American Mathematical Society, Providence (1993)

19. Havel, T., Wüthrich, K.: An evaluation of the combined use of nuclear magnetic resonance and distance geometry for the determination of protein conformations in solution. J. Mol. Biol. 182(2), 281-294 (1985)

20. Henneberg, L.: Die Graphische Statik der starren Systeme. Teubner, Leipzig (1911)

21. Heron: Metrica, vol. I. Alexandria ( 50AD)

22. Jackson, B., Jordán, T.: Graph theoretic techniques in the analysis of uniquely localizable sensor networks. In: Mao, G., Fidan, B. (eds.) Localization Algorithms and Strategies for Wireless Sensor Networks: Monitoring and Surveillance Techniques for Target Tracking, pp. 146-173. IGI Global, Hershey (2009)

23. Krislock, N., Wolkowicz, H.: Explicit sensor network localization using semidefinite representations and facial reductions. SIAM J. Optim. 20, 2679-2708 (2010)

24. Lavor, C., Firer, M., Martínez, J., Liberti, L.: Preface to special issue on many faces of distances. Int. Trans. Oper. Res. 23(5), 199-200 (2016)

25. Lavor, C., Liberti, L., Lodwick, W., da Costa, T.M.: An Introduction to Distance Geometry applied to Molecular Geometry. Springer Briefs. Springer, New York (2017)

26. Lavor, C., Liberti, L., Maculan, N.: Computational experience with the molecular distance geometry problem. In: Pintér, J. (ed.) Global Optimization: Scientific and Engineering Case Studies, pp. 213-225. Springer, Berlin (2006)

27. Lavor, C., Liberti, L., Maculan, N.: Molecular distance geometry problem. In: Floudas, C., Pardalos, P. (eds.) Encyclopedia of Optimization, 2nd edn, pp. 2305-2311. Springer, New York (2009)

28. Lavor, C., Liberti, L., Maculan, N., Mucherino, A.: Recent advances on the discretizable molecular distance geometry problem. Eur. J. Oper. Res. 219, 698-706 (2012)

29. Lavor, C., Liberti, L., Mucherino, A.: The interval Branch-and-Prune algorithm for the discretizable molecular distance geometry problem with inexact distances. J. Glob. Optim. 56, 855-871 (2013)

30. Lavor, C., Mucherino, A., Liberti, L., Maculan, N.: On the computation of protein backbones by using artificial backbones of hydrogens. J. Glob. Optim. 50, 329-344 (2011)

31. Liberti, L., Lavor, C.: Six mathematical gems in the history of distance geometry. Int. Trans. Oper. Res. 23, 897-920 (2016)

32. Liberti, L., Lavor, C.: Euclidean Distance Geometry: An Introduction. Springer, New York (2017)

33. Liberti, L., Lavor, C., Maculan, N.: A branch-and-prune algorithm for the molecular distance geometry problem. Int. Trans. Oper. Res. 15, 1-17 (2008)

34. Liberti, L., Lavor, C., Maculan, N., Mucherino, A.: Euclidean distance geometry and applications. SIAM Rev. 56(1), 3-69 (2014)

35. Liberti, L., Lavor, C., Mucherino, A., Maculan, N.: Molecular distance geometry methods: from continuous to discrete. Int. Trans. Oper. Res. 18, 33-51 (2010)

36. Maxwell, J.: On reciprocal figures and diagrams of forces. Philos. Mag. 27(182), 250-261 (1864)

37. Maxwell, J.: On the calculation of the equilibrium and stiffness of frames. Philos. Mag. 27(182), 294-299 (1864)

38. Menger, K.: New foundation of Euclidean geometry. Am. J. Math. 53(4), 721-745 (1931)

39. Mucherino, A., Lavor, C., Liberti, L., Maculan, N. (eds.): Distance Geometry: Theory, Methods, and Applications. Springer, New York (2013)

40. Mucherino, A., Liberti, L., Lavor, C.: MD-jeep: an implementation of a branch-and-prune algorithm for distance geometry problems. In: Fukuda, K., van der Hoeven, J., Joswig, M., Takayama, N. (eds.) Mathematical Software, LNCS, vol. 6327, pp. 186-197. Springer, New York (2010)

41. Singer, A.: Angular synchronization by eigenvectors and semidefinite programming. Appl. Comput. Harmon. Anal. 30, 20-36 (2011)

42. Skiena, S., Sundaram, G.: A partial digest approach to restriction site mapping. Bull. Math. Biol. 56(2), 275-294 (1994)

43. Thorpe, M., Duxbury, P. (eds.): Rigidity Theory and Applications. Fundamental Materials Research. Springer, New York (2002)

44. Yemini, Y.: The positioning problem-a draft of an intermediate summary. In: Proceedings of the Conference on Distributed Sensor Networks, pp. 137-145. Carnegie-Mellon University, Pittsburgh (1978) 